\title{
Source localization with an advanced gravitational wave detector network
}

\author{
Stephen Fairhurst \\ Cardiff School of Physics and Astronomy, Cardiff University, Queens Buildings, \\ The Parade, Cardiff. CF24 3AA \\ E-mail: Stephen.Fairhurst@astro.cf.ac.uk
}

\begin{abstract}
We derive an expression for the accuracy with which sources can be localized using a network of gravitational wave detectors. The result is obtained via triangulation, using timing accuracies at each detector and is applicable to a network with any number of detectors. We use this result to investigate the ability of advanced gravitational wave detector networks to accurately localize signals from compact binary coalescences. We demonstrate that additional detectors can significantly improve localization results and illustrate our findings with networks comprised of the advanced Laser Interferometer Gravitational-wave Observatory (LIGO), advanced Virgo and Large Cryogenic Gravitational Wave Telescope (LCGT). In addition, we evaluate the benefits of relocating one of the advanced LIGO detectors to Australia.
\end{abstract}

\section{Introduction}

The era of advanced gravitational wave detectors [1, 2, 3] is rapidly approaching, and with it the prospect of the regular observation of gravitational wave signals. To fully exploit these gravitational wave observations, it will be critically important to correlate them whenever possible with electromagnetic counterparts. For example, observation of gravitational waves coincident with Gamma Ray Bursts will aid in determining the nature of the progenitor [4]; the gravitational wave signal from core-collapse supernovae may carry information about the supernova engine [5]; gravitational and electromagnetic observations of binary coalescence will provide independent measures of the distance and redshift to the source and consequently enable precision tests of cosmology [6. One method of performing multi-messenger observations will be to accurately and rapidly localize the source through gravitational wave observations and then use electromagnetic observatories to follow up on the event. Indeed, an ambitious project to follow up gravitational wave candidates in a host of electromagnetic telescopes is already underway [7. A single gravitational wave detector gives virtually no directional information for a short duration signal. Consequently, localization requires a network of detectors and the primary tool for localization is triangulation between a signal observed at several sites $[8]$.

A number of electromagnetic telescopes are being designed, built and operated specifically to search for transient phenomena. These include Palomar Transient Factory [9, Pan Starrs [10, SkyMapper [11, LOFAR [12] and many more. These observatories will have a field of view of around ten square degrees. There are plausible astrophysical mechanisms that produce both gravitational waves and electromagnetic 
signals in various frequencies, for example gamma ray, x-ray, optical and radio (see, e.g. 4, 13, 14, 15). Thus, providing a good estimate of the localization capability of the gravitational wave network will help inform the follow-up searches which are undertaken. In addition, if the source can be localized to a region which contains only one, or possibly a few, galaxies then a narrow field telescope could be used to image the galaxy in question [16].

In this paper, we investigate the accuracy with which gravitational wave sources can be localized using a network of advanced detectors. A network comprising more detectors, particularly if they are at widely separated sites, gives better localization. Thus, the recent announcement of funding for the construction of the Japanese LCGT [2] is particularly welcome. This detector will augment the advanced LIGO (aLIGO) detectors [1] to be installed at sites at Hanford, WA and Livingston, LA in the USA and the advanced Virgo [3] detector to be installed in Cascina, Italy and provide a four site network. Furthermore, there is significant interest in installing one of the three advanced LIGO detectors at the Gingin site in Australia [17, 18, providing a realistic prospect for a five site network of advanced gravitational wave detectors.

The layout of the paper is as follows. In Section 2 we extend the framework presented in 8 to provide a simple, geometrical expression for the localization region obtained with any number of detectors. Then, in Section 3, we examine the capacity of the various networks to localize sources. The formalism is equally applicable to any transient source. However, for simplicity we present a sample of results for binary neutron star (BNS) mergers. As noted in [8, the results for both neutron star-black hole and binary black hole mergers with a total mass below around $10 M_{\odot}$ will be comparable.

\section{Sky Localization from Triangulation}

For the purposes of this paper, we assume that localization is achieved entirely by triangulation of the source by the network of gravitational wave detectors. In reality, there is additional information available in the waveform; for instance the fact that the gravitational waveform consists of only two polarizations can be used to coherently combine the data streams and improve localization [19, 20, 21. Indeed, even the non-observation of an event in one detector in the network can be used to assist with localization. Generically, these effects will serve to improve the localization of events. In addition, there will be numerous systematic uncertainties in estimating the localization of signals; for example imperfect calibration of the detectors and uncertainties in the emitted gravitational waveform. We will not investigate these in great detail, but merely note that systematic errors are expected to be smaller than statistical uncertainties for all but the loudest sources 8 .

\subsection{Timing}

The accuracy with which the timf $\mathrm{f}$ a gravitational wave signal passed a given detector can be determined is given by $[\underline{8}]$

$$
\sigma_{t}=\frac{1}{2 \pi \rho \sigma_{f}} .
$$

$\ddagger$ For an extended signal such as a binary coalescence, this should be regarded as a fiducial time, for example the time at which the frequency of the signal passes through $100 \mathrm{~Hz}$.

$\S$ A similar expression was obtained previously in [22]. 


\begin{tabular}{|l|c|c|c|c|c|}
\hline Detector & $\begin{array}{c}\text { Horizon } \\
(\mathrm{Mpc})\end{array}$ & $\begin{array}{c}\text { Range } \\
(\mathrm{Mpc})\end{array}$ & $\begin{array}{c}\bar{f} \\
(\mathrm{~Hz})\end{array}$ & $\begin{array}{c}\sigma_{f} \\
(\mathrm{~Hz})\end{array}$ & $\begin{array}{c}\sigma_{t} \text { at } \rho=8 \\
(\mathrm{~ms})\end{array}$ \\
\hline aLIGO NOSRM [24] & 360 & 160 & 65 & 43 & 0.46 \\
aLIGO BNS 24] & 490 & 215 & 110 & 106 & 0.19 \\
Advanced Virgo [3] & 350 & 155 & 120 & 100 & 0.20 \\
LCGT 2] & 365 & 160 & 100 & 88 & 0.22 \\
\hline
\end{tabular}

Table 1. Currently projected advanced detector sensitivity for BNS systems. The sensitivity of the detector is encoded in the BNS horizon (distance at which an optimally oriented and located signal gives an SNR of 8) and the BNS range (the volume and orientation averaged distance at which a BNS gives SNR 8). The horizon is a factor of 2.26 larger than the range. The mean frequency $\bar{f}$ and frequency bandwidth give the frequency range in which the detector is sensitive to the signal. The frequency bandwidth $\sigma_{f}$ determines the timing width $\sigma_{t}$ at a given SNR through (1). The two sets of advanced LIGO numbers correspond to no signal recycling (NOSRM) and BNS optimized (BNS) configurations.

Timing accuracy is inversely proportional to both the signal-to-noise ratio (SNR) $\rho$ and effective bandwidth $\sigma_{f}$ of the source, defined as

$$
\begin{aligned}
\rho^{2} & =4 \int_{0}^{\infty} \frac{|h(f)|^{2}}{S(f)} d f, \\
\sigma_{f}^{2} & =\left(\frac{4}{\rho^{2}} \int_{0}^{\infty} d f \frac{f^{2}|h(f)|^{2}}{S(f)}\right)-\left(\frac{4}{\rho^{2}} \int_{0}^{\infty} d f \frac{f|h(f)|^{2}}{S(f)}\right)^{2},
\end{aligned}
$$

where $S(f)$ denotes the one sided noise power spectral density of the detector. The approximations used to obtain this formula break down at low SNR, where second order effects become important 23. At an SNR of 8, which we consider in several later examples, this is about a $10 \%$ effect.

In Table1 we provide the currently projected sensitivities, frequency bandwidths and timing accuracies for the various advanced gravitational wave detectors. Numerous configurations have been proposed for the advanced detectors [1], and it is likely that several will be used over the lifetime of the detectors. To illustrate the differences, we consider two aLIGO configurations, one with no signal recycling mirror (NOSRM) which is likely to be an early configuration, and one optimized for BNS detection (BNS) which may be used in later science runs. The anticipated noise curves represent the incoherent sum of the principal noise sources as currently understood. There will be, in addition, technical noise sources. The sensitivities in Table 1 are not the guaranteed performance of advanced detectors, but a good guide to the anticipated sensitivity.

For the most part, advanced detectors will be sensitive to an optimally oriented and located BNS to a horizon distance of around $360 \mathrm{Mpc}$, although the BNS optimized aLIGO configuration provides a horizon of almost $500 \mathrm{Mpc}$. The frequency bandwidth of a BNS signal in the detectors will be around $100 \mathrm{~Hz}$, leading to a timing accuracy of $0.2 \mathrm{~ms}$ at an SNR of 8 . The aLIGO NOSRM configuration has a noise curve which rises more sharply at high frequency. Consequently, it has a significantly smaller bandwidth and a timing accuracy of almost $0.5 \mathrm{~ms}$ at SNR 8 , more than a factor of 2 larger than the other configurations listed.

For the remainder of this paper, we make the simplifying assumption that all the advanced detectors have the same sensitivity and bandwidth. Our standardized advanced detector will have a BNS horizon of $360 \mathrm{Mpc}$ (corresponding to a sky and 
orientation averaged $B N S$ range of $160 \mathrm{Mpc}$ at SNR 8) and a bandwidth of $100 \mathrm{~Hz}$. It is relatively straightforward to scale the results to other parameter choices using Equation (11).

\subsection{Localization}

The measured time of arrival of a signal in a network of detectors can be used to reconstruct the source location. However, consideration of the observed amplitudes and phases of the signal at all detectors in the network is critical for extracting the full set of parameters, including the distance to the binary and its orientation [25, 20. For our purposes a gravitational wave signal is described by the location $\mathbf{R}$ of the source and the time $T_{o}$ at which the signal passes through the center of the earth. Since our primary focus is localization, we take $\mathbf{R}$ to be a unit vector describing only the position of the source and not its distance.

The time at which the signal passes through detector $i$ is given by

$$
T_{i}=T_{o}-\mathbf{R} \cdot \mathbf{d}_{i},
$$

where $\mathbf{d}_{i}$ encodes the separation between detector $i$ and the center of the earth (expressed in seconds). The distribution of the measured arrival times $t_{i}$ in the various detectors, given the actual arrival times $T_{i}$, is given by

$$
p\left(t_{i} \mid T_{i}\right)=\prod_{i} \frac{1}{\sqrt{2 \pi} \sigma_{i}} \exp \left[\frac{-\left(t_{i}-T_{i}\right)^{2}}{2 \sigma_{i}^{2}}\right],
$$

where the timing accuracy $\sigma_{i}$ for each detector is given by Equation (1), and we assume that timing errors are Gaussian distributed.

Measurements of the arrival times in each detector can be used to construct a posterior probability distribution for the source's sky location $\mathbf{R}$. This is done by applying Bayes' theorem to obtain the posterior distribution for the actual arrival times as a function of the observations as

$$
p\left(T_{i} \mid t_{i}\right) \propto p\left(T_{i}\right) \exp \left[-\sum_{i} \frac{\left(t_{i}-T_{i}\right)^{2}}{2 \sigma_{i}^{2}}\right] .
$$

The posterior distribution is the product of the prior distribution $p\left(T_{i}\right)$ for the arrival times with the likelihood. Since we are interested in obtaining a distribution for the sky location of the event, we would like to re-express (5) in terms of $\mathbf{R}$. To do so, we introduce the measured sky position $\mathbf{r}$ and arrival time $t_{o}$ (in analogy to equation (3)) as

$$
t_{i}=t_{o}-\mathbf{r} \cdot \mathbf{d}_{i} .
$$

Strictly, for an event observed in multiple detectors, the set of equations (6) for $t_{o}$ and $\mathbf{r}$ may be over-determined and not allow any solution. Although the observed arrival times of a signal should be consistent with a specific geocentric arrival time and sky location, in practice, they will not be precisely consistent due to measurement errors. In the appendix, we discuss techniques which can be used to find best fit parameters for $t_{o}$ and $\mathbf{r}$. However, for the remainder of this section, we simply assume that we can eliminate $t_{i}$ in favour of $\mathbf{r}$ and $t_{o}$.

We also make use of (3) to eliminate $T_{i}$ from (5) in favour of $\mathbf{R}$ and $T_{o}$. The prior distributions are naturally taken to be uniform over the sphere (for $\mathbf{R}$ ) and uniform 
in time (for $T_{o}$ ). Finally, after marginalizing over $T_{o}$, the posterior distribution for $\mathbf{R}$ is

$$
p(\mathbf{R} \mid \mathbf{r}) \propto p(\mathbf{R}) \exp \left[-\frac{1}{2}(\mathbf{r}-\mathbf{R})^{T} \mathbf{M}(\mathbf{r}-\mathbf{R})\right] .
$$

The matrix $\mathbf{M}$, describing the localization accuracy, is given by

$$
\mathbf{M}=\frac{1}{\sum_{k} \sigma_{k}^{-2}} \sum_{i, j} \frac{\mathbf{D}_{i j} \mathbf{D}_{i j}^{T}}{2 \sigma_{i}^{2} \sigma_{j}^{2}}
$$

where $\mathbf{D}_{i j}=\mathbf{d}_{i}-\mathbf{d}_{j}$. A detailed derivation of this result is provided in the Appendix. A similar result has been obtained previously in 26 .

Equation (7) provides a simple extension to an arbitrary number of detectors of the two and three detector result given in 8. The localization expression has all the features we would expect, specifically: localization only depends upon the difference in arrival time between the various detectors; localization is improved by extending the baseline between detectors and by better timing accuracy in the detectors; the timing measurement in a pair of detectors can only serve to restrict the location of the source in the direction parallel to the detector separation. The origin of the normalization pre-factor for the matrix $\mathbf{M}$ arises due to the marginalization over the geocentric arrival time $T_{o}$ based upon timing information at all detectors in the network.

The matrix $\mathbf{M}$ is symmetric and can be diagonalized to obtain three orthogonal eigen-directions $\left(\hat{e}_{x}, \hat{e}_{y}, \hat{e}_{z}\right)$ with localization accuracies $\sigma_{x}, \sigma_{y}, \sigma_{z}$ respectively. Thus, the posterior distribution for the sky location is

$$
p(\mathbf{R} \mid \mathbf{r}) \propto p(\mathbf{R}) \exp \left[-\frac{1}{2}\left(\frac{(x-X)^{2}}{\sigma_{x}^{2}}+\frac{(y-Y)^{2}}{\sigma_{y}^{2}}+\frac{(z-Z)^{2}}{\sigma_{z}^{2}}\right)\right]
$$

where $\mathbf{R}=(X, Y, Z)$ are the co-ordinates of the source in the network eigen-basis, and $\mathbf{r}=(x, y, z)$ describes the measured location. The widths $\sigma_{x}, \sigma_{y}, \sigma_{z}$ encapsulate the ability of the network to localize sources.

The sky position $\mathbf{R}=(X, Y, Z)$ is restricted to lie on the unit sphere, and we must take this into account to obtain localization regions. Geometrically, equation (9) describes ellipsoids of constant likelihood which intersect the unit sphere to give the localization distribution as an ellipse on the sky. In most cases, the source will be localized to a small enough patch of this sphere that ignoring its curvature is a good approximation. In this case, we project $\mathbf{M}$ onto directions orthogonal to $\mathbf{r}$ using the projection

$$
\mathbf{P}(\mathbf{r})=\mathbf{I}-\mathbf{r r}^{T}
$$

where $\mathbf{I}$ is the identity matrix. This gives

$$
\mathbf{M}(\mathbf{r})=\mathbf{P}(\mathbf{r}) \mathbf{M} \mathbf{P}(\mathbf{r}) \text {. }
$$

The eigenvectors and corresponding eigenvalues of $\mathbf{M}(\mathbf{r})$ describe the two dimensional localization ellipse for the source. The best localization arises when the projection preserves the two smallest $\sigma$ values, and the worst when it keeps the two largest. In all cases, the source is localized with a probability $p$ within an area

$$
\operatorname{Area}(p) \approx 2 \pi \sigma_{1} \sigma_{2}[-\ln (1-p)],
$$

where $\sigma_{1}$ and $\sigma_{2}$ are the localization accuracies of the eigen-directions of the projected matrix $\mathbf{M}(\mathbf{r})$. 
When the network is degenerate, one or more of the $\sigma_{i}$ is infinite and the constant likelihood ellipsoids have infinite extent in one or more direction. For a three detector network, there is one degenerate direction perpendicular to the plane of the detectors and the surfaces become cylinders with infinite extent in that direction. Intersecting the cylinder with the unit sphere gives two localization regions, one above and the other below the plane formed by the detectors. Generically, this degeneracy can be broken by consideration of the observed amplitudes in the three detectors, and we assume this can be done. Taking the normal to the detector plane to be in the $z$ direction, the localization area for a three site network can be re-expressed in terms of $\sigma_{x}$ and $\sigma_{y}$ as

$$
\operatorname{Area}(p) \approx 2 \pi \sigma_{x} \sigma_{y}[-\ln (1-p)] / \cos \theta,
$$

where $\theta$ is the angle between the z-direction and the source. If the source lies in (or close to) the $x-y$ plane, then it cannot be well localized, and the approximation used to obtain (13) breaks down. In this case we must go back to the full distribution to derive the localization region. For a source lying in the $x$-direction, we obtain

$$
\operatorname{Area}(p) \approx 2 \pi \sigma_{y} \sqrt{2 \sigma_{x}}[-\ln (1-p)] .
$$

For a two site network, only $\sigma_{x}$ is finite and the source location is restricted to a ring on the sky. The minimal area of the sky containing the source with a fixed probability $p$ is independent of the source location and given as:

$$
\operatorname{Area}(p) \approx 4 \pi \frac{\sqrt{2} \sigma_{x} \operatorname{erf}^{-1}(p)}{D},
$$

where $D$ is the separation between the two detectors.

We make repeated use of these localization expressions (12) in the remainder of the paper where we examine the localization capabilities of various detector networks.

\section{Advanced detector networks}

Using the results of Section 2, we can evaluate the sky coverage and localization provided by various advanced detector networks. We take the baseline network to be the aLIGO network with two detectors in Hanford, WA (denoted $\mathrm{H}$ ) and one in Livingston, LA (denoted L) supplemented with any number of:

- the Japanese LCGT detector (denoted J) to be located at the Kamioka mine,

- the advanced Virgo detector (denoted V) at Cascina, Italy,

- the installation of an aLIGO detector at the Gingin site in Australia (denoted A), and consequently only a single aLIGO detector at Hanford.

This provides a set of eight advanced detector networks whose sensitivity and localization ability can be compared. For simplicity, we take all advanced detectors to have a BNS horizon of $360 \mathrm{Mpc}$ and a frequency bandwidth of $100 \mathrm{~Hz}$ (corresponding to a timing accuracy of $0.2 \mathrm{~ms}$ at SNR 8 ). We perform three different comparison studies. First, in Section 3.1 we consider a source observed in all detectors in the network with an SNR of 8. Then, in Section 3.2, we consider a set of face on binaries at a fixed distance from various sky positions. Finally, in Section 3.3, we consider an astrophysically distributed population of sources. A subset of these results were used in presenting the scientific case for installing one of the advanced LIGO detectors in Australia [17, 18]. 


\begin{tabular}{|c|c|c|c|}
\hline Network & Localization $\left(^{\circ}\right)$ & Best Area $\left(\mathrm{deg}^{2}\right)$ & Worst Area $\left(\mathrm{deg}^{2}\right)$ \\
\hline HHL & $(1.4,-,-)$ & 1700 & 1700 \\
AHL & $(0.4,1.7,-)$ & 8 & 150 \\
\hline HHJL & $(0.5,2.0,-)$ & 14 & 220 \\
AHJL & $(0.3,0.7,4.8)$ & 4 & 51 \\
\hline HHLV & $(0.5,1.4,-)$ & 10 & 150 \\
AHLV & $(0.4,0.6,1.7)$ & 3 & 14 \\
\hline HHJLV & $(0.5,0.5,2.9)$ & 4 & 22 \\
AHJLV & $(0.3,0.6,0.7)$ & 3 & 6 \\
\hline
\end{tabular}

Table 2. Localization accuracy in networks of detectors. We assume a BNS signal observed at SNR 8 in each detector or, equivalently, a timing uncertainty of $0.2 \mathrm{~ms}$ in each detector. The localization widths correspond to values of $\left(\sigma_{x}, \sigma_{y}, \sigma_{z}\right)$ from equation (9) and have been expressed in degrees. For the two site HHL network, localization is only possible in one direction, while for the three site networks there is a degenerate direction perpendicular to the plane of the network. The best and worst case areas are 90\% confidence areas calculated using equations (12 15).

\subsection{Signal at a fixed SNR}

Table 2 summarizes the localization results for BNS sources observed with an SNR of 8 in all detectors. It is rather unlikely that a signal will be observed with the same SNR in all detectors in a network, due to the different antenna patterns of the detectors. However, this simple scenario provides a straightforward method of comparing different networks. For each of the eight advanced detector networks, we give the localization widths $\left(\sigma_{x}, \sigma_{y}, \sigma_{z}\right)$ from equation (9) as well as the smallest and largest areas of the $90 \%$ confidence localization regions.

The advanced LIGO network (HHL) comprises only two sites and, as expected, is unable to provide satisfactory localization for any source. The three site networks (AHL, HHJL, HHLV) provide good localization in some directions but degeneracies remain for sources close to the plane of the detectors. Although it comprises only three detectors, the AHL network provides a particularly small "best case" localization region due to the long baselines between the US and Australia. The results for the aLIGO-Virgo (HHLV) network are somewhat better than aLIGO-LCGT (HHJL), even though the baselines are comparable. The Livingston, Hanford and LCGT are close to co-linear, providing one good localization direction and one relatively poor one.

The addition of a fourth site to the network (e.g. AHJL, AHLV, HHJLV) results in a significant improvement in the "worst case" localization. However, there remain some directions with relatively poor localization for the AHJL and HHJLV networks as these sites are nearly co-planar. The five site network AHJLV provides good source localization over the full sky.

\subsection{Source at a fixed distance}

Here, we consider localization for a face on BNS coalescence at a distance of $160 \mathrm{Mpc}$ at different locations in the sky. The expected SNR in each detector depends upon its sensitivity to the source direction. Consequently, we introduce a simple criterion to classify signals as detectable: the expected SNR in the network must exceed 12, and the individual SNR at two or more sites must exceed 5. These thresholds are motivated by results of LIGO-Virgo searches [27, 28, where an SNR threshold of 5.5 

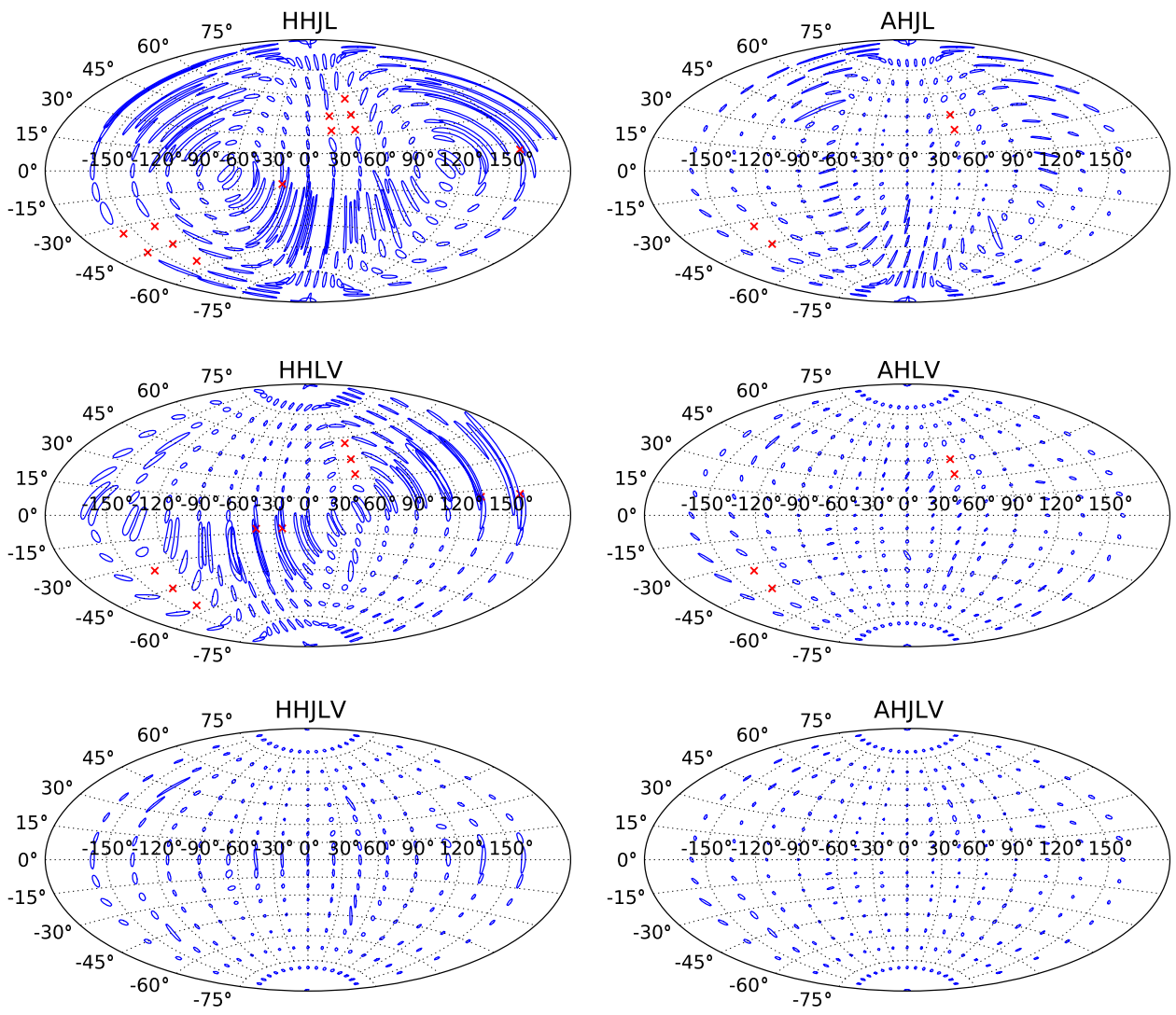

Figure 1. The localization accuracy for face on BNS at $160 \mathrm{MPc}$ in various networks of advanced detecors. The ellipses contain the $90 \%$ localization regions for sources from varioius points in the sky. A $\times$ is plotted at points where the network would not confidently detect the system. The plots show the localization for six different networks: Hanford-Hanford-LCGT-Livingston (HHJL); Australia-Hanford-LCGT-Livingston (AHJL); Hanford-Hanford-LivingstonVirgo (HHLV); Australia-Hanford-Livingston-Virgo (AHLV); Hanford-HanfordLCGT-Livingston-Virgo (HHJLV); Australia-Hanford-LCGT-Livingston-Virgo (AHJLV).

was used in each detector, and the loudest background events had a combined SNR of around 10. For those signals which are classified as detectable, the localization ellipse is calculated using the expressions given in Section 2 .

Figure 1 shows the localization ellipses for the four and five detector networks considered in Section 3.1 1 The results are consistent with those in Section 3.1. A three site network provides good localization in some regions of the sky, but close to the plane of the detectors the localization ellipses become extended. Furthermore, there are regions of the sky where the signal is deemed undetectable by our criteria. The addition of a fourth site breaks the three site degeneracy and significantly improves localization, particularly for the worst cases. However, there remain some slightly extended regions for the AHJL and HHJLV networks which lie close to the approximate

\| Similar results for a different source model have been presented in [26]. 


\begin{tabular}{|c|c|c|c|c|c|}
\hline Network & Detectable Sources & \multicolumn{4}{|c|}{ Sources Localized within } \\
& & $1 \mathrm{deg}^{2}$ & $5 \mathrm{deg}^{2}$ & $10 \mathrm{deg}^{2}$ & $20 \mathrm{deg}^{2}$ \\
\hline HHL & 59 & 0 & 0 & 0 & 0 \\
AHL & 59 & 0.4 & 5 & 13 & 30 \\
\hline HHJL & 85 & 0.2 & 2 & 5 & 14 \\
AHJL & 85 & 1 & 14 & 36 & 59 \\
\hline HHLV & 83 & 0.4 & 5 & 13 & 35 \\
AHLV & 84 & 2 & 21 & 48 & 76 \\
\hline HHJLV & 112 & 2 & 19 & 47 & 77 \\
AHJLV & 114 & 3 & 34 & 84 & 111 \\
\hline
\end{tabular}

Table 3. Sensitivity and localization capability of various different advanced detector networks to a population of BNS signals. The number of signals is normalized so that there are 40 signals observed in any single detector above $\mathrm{SNR}=8$. This corresponds to the annual astrophysical rate estimate presented in 29]. For each detected signal, we calculate the $90 \%$ localization area and count those which are localized within 1, 5, 10 and $20 \mathrm{deg}^{2}$.

plane formed by the four sites. The five site network AHJLV shows excellent resolution over the entire sky.

\subsection{A Population of Coalescing Binaries}

The rate of binary coalescences in spiral galaxies is expected to follow star formation rate or, equivalently, the blue light luminosity of the galaxies [29, and there may well be additional contributions from elliptical galaxies and globular clusters [30. However, with detector sensitivities extending to hundreds of Mpc, it is reasonable to assume a population which is uniformly distributed in volume. Additionally, there is no reason to expect a preferred orientation of binary systems in the universe, so we take a uniform distribution of binary orientations. We use these distributions to simulate the parameters of a large number of potential signals and for each signal determine whether is is "detectable" in a given network, using the same conditions as previously. For signals which are detected, we calculate the $90 \%$ localization area from equations (12,[15).

Table 3 summarizes the results of the simulation. For each detector network, the expected number of detectable signals as well as the number localizable within 1, 5, 10 and $20 \mathrm{deg}^{2}$ is given. The numbers are normalized to give 40 signals with SNR greater than 8 in a single detector, in accordance with the "realistic" estimate of the annual astrophysical rate 29. However, there is at least an order of magnitude uncertainty in the rate of BNS signals. Additionally, some relatively simplistic assumptions have been made for the detection threshold. Thus, the results in Table 3 should be taken as illustrative: significant difference between network performance are meaningful, but the actual values should not be taken too literally.

The results for a population of sources again provide a strong case for the construction of as many detectors at different sites as possible. As well as an increase in the absolute number of observable sources, additional detectors greatly increase the fraction of sources which can be well localized. For the HHL network no sources will be localized within $20 \mathrm{deg}^{2}$. With the introduction of a third site (AHL, HHLV, HHJL) a significant fraction (20 to 50\%) of sources are localized within $20 \mathrm{deg}^{2}$, and the loudest signals may be localized within $5 \mathrm{deg}^{2}$. The addition of a fourth site to 

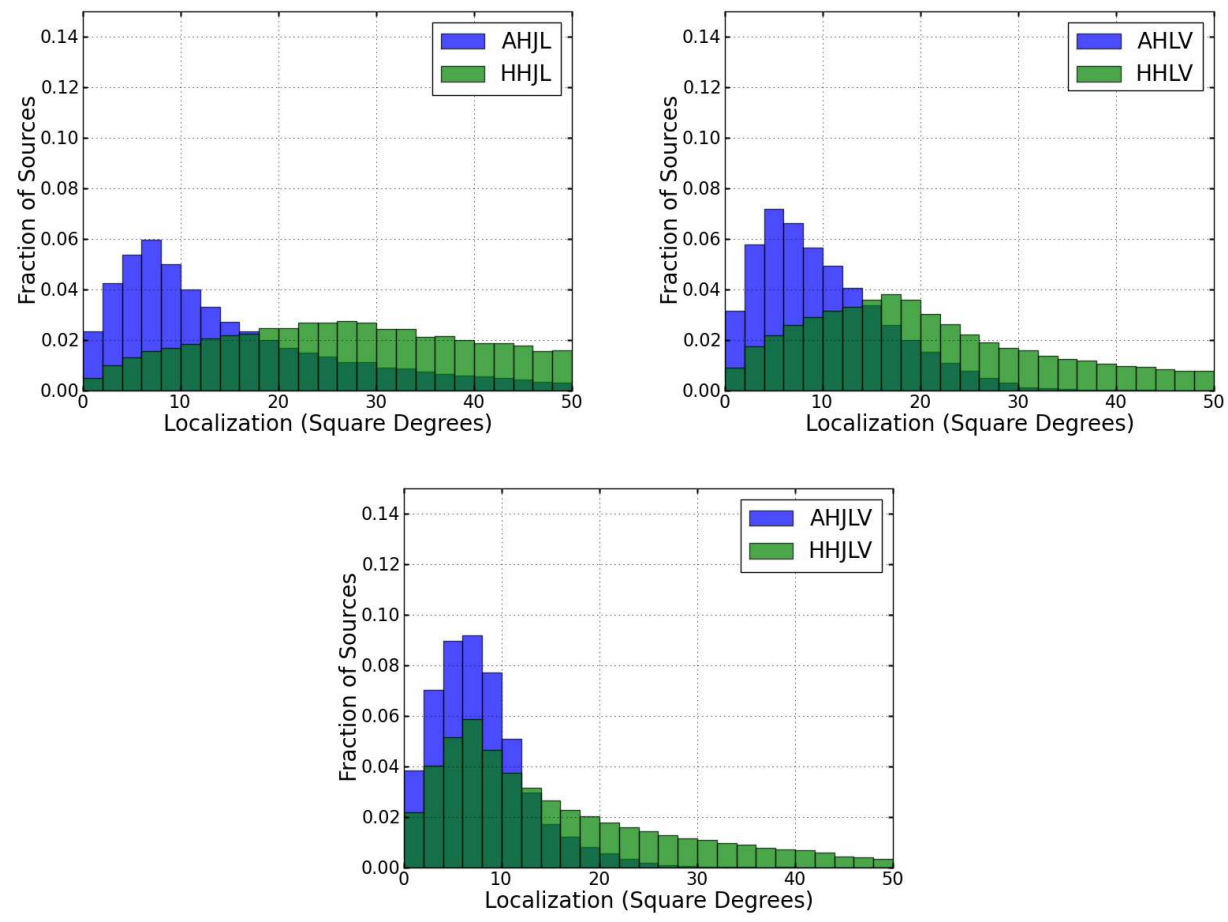

Figure 2. Distributions of localization accuracy for various networks

the network (AHLV, AHJL, HHJLV) further improves localization, with the majority of signals localized within $20 \mathrm{deg}^{2}$, and as many as $20 \%$ to within $5 \mathrm{deg}^{2}$. The five site network provides the most remarkable results, with virtually all signals localized within $20 \mathrm{deg}^{2}$, a third within $5 \mathrm{deg}^{2}$ and the loudest to within a square degree.

Figure 2 provides a graphical representation of the same data, showing the localization distribution areas for different networks. In all cases, the addition of more sites improves the localization. For the networks involving an Australian detector, the peak of the localization distribution occurs between 5 and $10 \mathrm{deg}^{2}$. This corresponds to the typical area of the wide field electromagnetic transient telescopes currently being operated or under construction [9, 10, 11, 12].

\section{Discussion}

We have obtained an expression for the localization accuracy of a gravitational wave signal in a network of detectors. The localization expression makes use of only timing information in the various detectors, with the timing uncertainty taken to be inversely proportional to both SNR and signal bandwidth. This extends the results of [8] to networks with any number of detectors. As expected, localization depends only upon the difference in arrival time between the various detectors, is improved by better timing accuracy in individual detector and longer baselines between detectors. The expressions presented here make numerous simplifying assumptions by, for example, treating the data as Gaussian and stationary, using only timing information for 
localization, taking the leading order contributions to the timing and localization distributions, neglecting systematic uncertainties in the waveform and instrumental calibration. Thus, while providing useful localization estimates, a real implementation of source localization would need to address many additional issues.

We have examined localization of BNS sources with a network of advanced gravitational wave detectors. It seems reasonable to assume that the first operational advanced detectors will be at the existing sites (LIGO Hanford, LIGO Livingston, Virgo) as much of the infrastructure, including the beam tubes, is already in place. Furthermore, there is a good chance that early science runs will be undertaken without a signal recycling mirror (the NOSRM configuration in Table11). This gives a frequency bandwidth of $40 \mathrm{~Hz}$ for a BNS signal, compared to around $100 \mathrm{~Hz}$ in the other configurations. Consequently, the localization accuracy of the network could initially be about a factor of five worse than the results presented in Section 3, meaning only the loudest signals will be well localized. However, there is the prospect for significant improvement with the installation of signal recycling to provide a more broadband spectrum and the addition of new sites (LIGO Australia, LCGT) to the network. A four site network operating with broadband sensitivity gives good sensitivity and localization accuracy over the majority of the sky, with the potential for up to half of detected BNS coalescences to be localized (with 90\% confidence) within $10 \mathrm{deg}^{2}$. The five site network provides outstanding coverage over the whole sky with the prospect of virtually every signal being localized within $20 \mathrm{deg}^{2}$ and a third within $5 \mathrm{deg}^{2}$. These localization areas are commensurate with the field of view of existing and planned electromagnetic transient observatories providing the realistic prospect for multi-messenger astronomy in the advanced detector era.

\section{Acknowledgements}

We have benefited from extensive discussions with many people within the LIGO Scientific and Virgo Collaborations. In particular we thank Duncan Brown, Jolien Creighton, Thomas Dent, Bangalore Sathyaprakash, Bernard Schutz, Patrick Sutton, John Veitch, Rai Weiss and the LIGO Australia committee. We thank Patrick Sutton for independently verifying the numbers used in Table 1. This research was made possible thanks to support from the Royal Society.

\section{Appendix A. Detailed derivation of the localization expression}

Here, we provide a detailed derivation of the localization expression (77) presented in Section 2.2. We begin with equation (4) which gives the probability distribution for the signal arrival times $T_{i}$ at the different detectors, given the measured times $t_{i}$,

$$
p\left(T_{i} \mid t_{i}\right) \propto p\left(T_{i}\right) \exp \left[-\sum_{i} \frac{\left(t_{i}-T_{i}\right)^{2}}{2 \sigma_{i}^{2}}\right] .
$$

We would like to eliminate the detector arrival times, both measured, $t_{i}$, and actual, $T_{i}$, in favour of geocentric arrival times and sky positions. It is straightforward to replace the actual arrival times $T_{i}$ with the actual sky location $\mathbf{R}$ (required to be a unit vector) and geocentric arrival time $T_{o}$ using

$$
T_{i}=T_{o}-\mathbf{R} \cdot \mathbf{d}_{i} .
$$


The observed arrival times will be subject to fluctuations due to noise in the detectors and the equivalent set of equations for $t_{o}$ and $\mathbf{r}$ may be over-determined and not allow any solution. Typically, a minimization technique is used to solve for the most consistent sky location and arrival time by minimizing [31]

$$
\chi^{2}=\sum_{i} \frac{\left(t_{i}-t_{o}+\mathbf{r} \cdot \mathbf{d}_{i}\right)^{2}}{\sigma_{i}^{2}} .
$$

In this appendix, we will not perform the full minimization, but instead restrict to the four dimensional surface spanned by $t_{o}, \mathbf{r}$, where we make no restriction on the modulus of $\mathbf{r}$. Of course, for a signal, we expect $|\mathbf{r}| \approx 1$. We choose not to enforce the exact equality $|\mathbf{r}|=1$ as it serves to simplify the following derivation. Working in the extended parameter space, the minimization can be performed using a weighted least squares approach. Differentiating (A.3) with respect to $t_{o}$ and $\mathbf{r}$ gives a set of linear equations

$$
\sum_{i} \frac{\left(t_{i}-\hat{t}_{o}+\hat{\mathbf{r}} \cdot \mathbf{d}_{i}\right)}{\sigma_{i}^{2}}=0 \text { and } \sum_{i} \frac{\mathbf{d}_{i}\left(t_{i}-\hat{t}_{o}+\hat{\mathbf{r}} \cdot \mathbf{d}_{i}\right)}{\sigma_{i}^{2}}=0
$$

which can be solved for the best fit values of the geocentric arrival time $\hat{t}_{o}$ and "sky location" $\hat{\mathbf{r}}$. These define a best fit set of arrival times

$$
\hat{t}_{i}=\hat{t}_{o}-\hat{\mathbf{r}} \cdot \mathbf{d}_{i} \text {. }
$$

The residual

$$
\chi_{\min }^{2}=\sum_{i} \frac{\left(t_{i}-\hat{t}_{i}\right)^{2}}{\sigma_{i}^{2}}
$$

encodes the goodness of fit of the timing data to the best fit values.

Having performed this partial maximization, we are in position to re-express the probability distribution (A.1) in terms of geocentric arrival times and sky locations. We begin by expanding

$$
\begin{aligned}
\sum_{i} \frac{\left(t_{i}-T_{i}\right)^{2}}{2 \sigma_{i}^{2}}= & \sum_{i} \frac{\left[\left(t_{i}-\hat{t}_{i}\right)+\left(\hat{t}_{i}-T_{i}\right)\right]^{2}}{2 \sigma_{i}^{2}} \\
= & \sum_{i} \frac{\chi_{\min }^{2}}{2}+\frac{1}{2 \sigma_{i}^{2}}\left[\left(\hat{t}_{o}-T_{o}\right)-(\hat{\mathbf{r}}-\mathbf{R}) \cdot \mathbf{d}_{i}\right]^{2} \\
& \quad-\frac{1}{\sigma_{i}^{2}}\left[t_{i}-\hat{t}_{o}+\hat{\mathbf{r}} \cdot \mathbf{d}_{i}\right]\left[\hat{t}_{o}-T_{o}-(\hat{\mathbf{r}}-\mathbf{R}) \cdot \mathbf{d}_{i}\right] .
\end{aligned}
$$

The first term, encoding the consistency of the measured arrival times, is constant (independent of $T_{o}$ and $\mathbf{R}$ ) and will therefore not affect the localization distribution (A.1). In addition, the third term vanishes. This follows directly from the expressions (A.4) used to determine the best fit parameters $\hat{t}_{o}$ and $\hat{\mathbf{r}}$. Consequently, the posterior distribution for sky location and arrival time can be expressed as

$$
p\left(\mathbf{R}, T_{o} \mid \hat{\mathbf{r}}, \hat{t}_{o}\right) \propto p\left(\mathbf{R}, T_{o}\right) \exp \left[-\sum_{i} \frac{\left[\left(\hat{t}_{o}-T_{o}\right)-(\hat{\mathbf{r}}-\mathbf{R}) \cdot \mathbf{d}_{i}\right]^{2}}{2 \sigma_{i}^{2}}\right]
$$

Next, we would like to marginalize over the arrival time $T_{o}$. The measurement uncertainties in the arrival time at the various detectors are typically fractions of a millisecond. Even in cases where the time of the event is well known from another 
astronomical observation, the arrival time of the gravitational wave signal will not be known with millisecond accuracy. Consequently, it is appropriate to take a uniform prior on the arrival time $T_{o}$, and also make the priors on arrival time and sky location independent. Then, the distribution (A.8) can be written as

$$
\begin{array}{r}
p\left(\mathbf{R}, T_{0} \mid \hat{\mathbf{r}}, \hat{t}_{o}\right) \propto p(\mathbf{R}) p\left(T_{o}\right) \exp \left[-\left(\hat{t}_{o}-T_{o}\right)^{2} \sum_{i} \frac{1}{2 \sigma_{i}^{2}}+\quad\right. \text { (A.9) } \\
\left.2\left(\hat{t}_{o}-T_{o}\right) \sum_{i} \frac{(\hat{\mathbf{r}}-\mathbf{R}) \cdot d_{i}}{2 \sigma_{i}^{2}}-\sum_{i} \frac{\left[(\hat{\mathbf{r}}-\mathbf{R}) \cdot \mathbf{d}_{i}\right]^{2}}{2 \sigma_{i}^{2}}\right] .
\end{array}
$$

In order to marginalize over $T_{o}$, we simply integrate over that variable. This is most easily done by completing the square in the expression (A.9), and performing the Gaussian integral. The resulting distribution for the sky location $\mathbf{R}$ is

$$
\begin{aligned}
p\left(\mathbf{R} \mid \hat{\mathbf{r}}, t_{o}\right)= & \int d T_{o} p\left(\mathbf{R}, T_{0} \mid \hat{\mathbf{r}}, \hat{t}_{o}\right) \\
\propto & p(\mathbf{R}) \exp \left[\left(\frac{-1}{2 \sum_{k} \sigma_{k}^{-2}}\right) \times\right. \\
& \left.\left(\sum_{i} \frac{\left[(\hat{\mathbf{r}}-\mathbf{R}) \cdot \mathbf{d}_{i}\right]^{2}}{\sigma_{i}^{2}} \sum_{j} \sigma_{j}^{-2}-\left[\sum_{i} \frac{(\hat{\mathbf{r}}-\mathbf{R}) \cdot \mathbf{d}_{i}}{\sigma_{i}^{2}}\right]^{2}\right)\right] \\
= & p(\mathbf{R}) \exp \left[-\frac{1}{2}(\hat{\mathbf{r}}-\mathbf{R})^{T} \mathbf{M}(\hat{\mathbf{r}}-\mathbf{R})\right]
\end{aligned}
$$

where we have introduced

$$
\mathbf{M}=\frac{1}{\sum_{k} \sigma_{k}^{-2}} \sum_{i, j} \frac{\mathbf{D}_{i j} \mathbf{D}_{i j}^{T}}{2 \sigma_{i}^{2} \sigma_{j}^{2}} \quad \text { and } \quad \mathbf{D}_{i j}=\mathbf{d}_{i}-\mathbf{d}_{j}
$$

This localization distribution is valid even the location vector $\hat{\mathbf{r}}$ is not unit. The restriction that the source originate from a point on the sky is imposed by the use of a prior distribution $p(\mathbf{R})$ which enforces $|\mathbf{R}|=1$. In the main body of the paper (Equations (7) and (8)), we have made the simplifying assumption that the measured times $t_{i}$ are consistent with a sky location $\mathbf{r}$, where $|\mathbf{r}|=1$. While not strictly necessary, it does serve to simplify the discussion of localization regions.

\section{References}

[1] Harry G M and the LIGO Scientific Collaboration 2010 Classical and Quantum Gravity 27 084006

[2] LCGT http://gw.icrr.u-tokyo.ac.jp/lcgt/

[3] Advanced Virgo http://wwwcascina.virgo.infn.it/advirgo/

[4] Nakar E 2007 Phys. Rept. 442 166-236

[5] Ott C D 2009 Classical and Quantum Gravity 26204015

[6] Schutz B F 1986 Nature 323 310-311

[7] Kanner J, Huard T L, Marka S, Murphy D C, Piscionere J, Reed M and Shawhan P 2008 Classical and Quantum Gravity 25184034

[8] Fairhurst S 2009 New J. Phys. 11123006

[9] Rau A et al. 2009 Publ. Astron. Soc. Pac. 121, 1334

[10] Pan-STARRS http://pan-starrs.ifa.hawaii.edu/public/

[11] Sky Mapper http://rsaa.anu.edu.au/skymapper/

[12] LOFAR http://www.lofar.org/

[13] Metzger B D et al. 2010 (Preprint 1001.5029) 
[14] Predoi V et al. 2010 Class. Quant. Grav. 27084018

[15] Chassande-Mottin E, Hendry M, Sutton P J and Marka S 2011 Gen. Rel. Grav. 43 437-464

[16] Nuttall L K and Sutton P J 2010 Phys. Rev. D82 102002

[17] Klimenko S, Saulson P, Sathyaprakash B, Fritschel P, Raab F, Weiss R and Finn L S 2010, LIGO Document LIGO-T1000251-v1, 2010 URL https://dcc.ligo.org/cgi-bin/DocDB/ShowDocument?docid=11604

[18] Cho A 2010 Science 3291003 URL/http://www. sciencemag.org/cgi/reprint/329/5995/1003.pdf

[19] Gürsel Y and Tinto M 1989 Phys. Rev. D 40 3884-3938

[20] Veitch J and Vecchio A 2010 Phys. Rev. D81 062003

[21] Röver C, Meyer R and Christensen N 2006 Classical and Quantum Gravity 234895

[22] Schutz B F 1991 Data processing, analysis, and storage for interferometric antennas The detection of gravitational waves ed Blair D (Cambridge, UK: Cambridge University Press) pp 406-452

[23] Vitale S and Zanolin M 2010 Phys. Rev. D82 (2010) 124065.

[24] The LIGO Scientific Collaboration, LIGO Document T0900288-v3, 2009 URL https://dcc.ligo.org/cgi-bin/DocDB/ShowDocument?docid=2974

[25] Pai A, Dhurandhar S and Bose S 2001 Phys. Rev. D64 042004

[26] Wen L and Chen Y 2010 Phys. Rev. D81 082001

[27] Abbott B P et al. (LIGO Scientific) 2009 Phys. Rev. D79 122001

[28] Abbott B P et al. (LIGO Scientific) 2009 Phys. Rev. D80 047101

[29] Abadie J et al. (LIGO Scientific) 2010 Class. Quant. Grav. 27173001

[30] O'Shaughnessy R, Kalogera V and Belczynski K 2010 Astrophys. J. 716 615-633

[31] Cavalier F et al. 2006 Phys. Rev. D74 082004 